\title{
The 3d Ising Spin Glass
}

\author{
Per Nordblad \\ Department of Materials Science, \\ Uppsala University, \\ Box 534, SE-751 21 Uppsala, Sweden
}

Received on 5 August, 2000

\begin{abstract}
Experimentally derived physical properties of $3 \mathrm{~d}$ Ising spin glasses are discussed and compared to what is anticipated from theory and simulations of model systems. It is found that the spin glass experiences a zero-field phase transition to a low-temperature spin-glass phase, that there is no phase transition in a magnetic field and that nonequilibrium and chaos effects often dominate the dynamics of the low-temperature glassy phase. These results are in agreement with findings from Monte Carlo simulations and with properties derived from the droplet model.
\end{abstract}

\section{Introduction}

3d spin glasses are described by the Edwards-Anderson (EA) model:

$$
H=-\frac{1}{2} \sum_{i, j} J_{i j} S_{i} S_{j}
$$

where $J_{i j}$ are random interactions with zero mean that can be chosen only as nearest neighbor interactions and $S_{i}$ and $S_{j}$ are spins on a regular three dimensional lattice [1]. This model Hamiltonian is realized by the $\mathrm{Fe}_{0.5} \mathrm{Mn}_{0.5} \mathrm{TiO}_{3}$ compound. The magnetic structure of the two parent compounds of this spin glass system is antiferromagnetic at low temperatures; the structure may be described by a hexagonal lattice with the spins directed along the c-axis of the crystals. In $\mathrm{FeTiO}_{3}$ the magnetic atoms are ferromagnetically coupled in the hexagonal planes but adjacent layers are antiferromagnetically coupled. In $\mathrm{MnTiO}_{3}$, on the other hand, neighboring $\mathrm{Mn}$ atoms are antiferromagnetically coupled within the layers. Mixing iron and manganese causes randomness and frustration, and at about equal concentration of Fe and $\mathrm{Mn}$ atoms, this system almost ideally reproduces a short range 3d EA spin glass[2]. Results from experiments on $\mathrm{Fe}_{0.5} \mathrm{Mn}_{0.5} \mathrm{TiO}_{3}$ will be used to discuss static and dynamic scaling analyses that suggest the existence of a finite temperature phase transition in zero field but no phase transition in a magnetic field. Nonequilibrium dynamics and aging phenomena [3] will be exemplified with representative data from other $3 \mathrm{~d}$ spin glass materials.

\section{Phase Transition}

Is there a spin glass phase in real $3 \mathrm{~d}$ Ising spin glasses? This key question may sound trivial to resolve experimentally, but has turned out to require extensive and careful studies on good model systems to find a reliable answer. We here recall static and dynamic scaling results on one and the same $\mathrm{Fe}_{0.5} \mathrm{Mn}_{0.5} \mathrm{TiO}_{3}$ single crystalline spin glass material $[4,5]$. Fig. 1 shows the in and out of phase components of the susceptibility of this material in a wide frequency range[5], and Fig. 2 shows results from ac susceptibility measurements in superposed dc magnetic fields [6]. Some essential features of spin glass dynamics can be seen in these figures, two of which are: (i) there is wide region in which consequences of a slowing down of the dynamics are evident in the ac-susceptibility; (ii) there is a substantial suppression of the susceptibility with field.

The observed frequency dependence of the susceptibility allows to safely distinguish a critical slowing-down behavior:

$$
\frac{\tau}{\tau_{0}}=t^{-z \nu}
$$

where $t$ is the reduced temperature $\left(\left(T-T_{g}\right) / T_{g}\right), \tau$ the relaxation time, $\tau_{0}$ the microscopic spin flip time and $z \nu$ the dynamic critical exponent. More and less sophisticated dynamic scaling analyses of the data of Fig. 1 all finally yield $z \nu=10 \pm 0.5$. [5]

Approaching the critical temperature the susceptibility, as is implied from Fig. 2, becomes more and more field dependent. This non-linear susceptibility can be accurately measured from, e.g., the dependence of the ac susceptibility on an increasing superposed dc field. Employing such data in static scaling analyses yields the following set of static critical exponents: $\gamma=4 \pm 0.5$, $\beta=0.5 \pm 0.1$ and $\delta=8 \pm 2$. [4] 


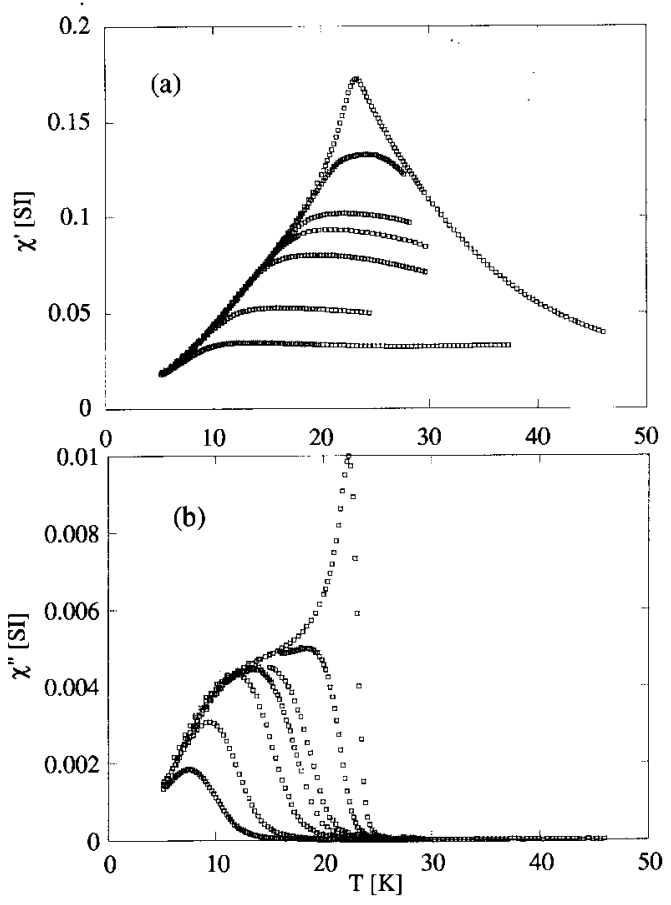

Figure 1. Ac-susceptibility of $\mathrm{Fe}_{0.5} \mathrm{Mn}_{0.5} \mathrm{TiO}_{3}$ at different frequencies from ref.[5].

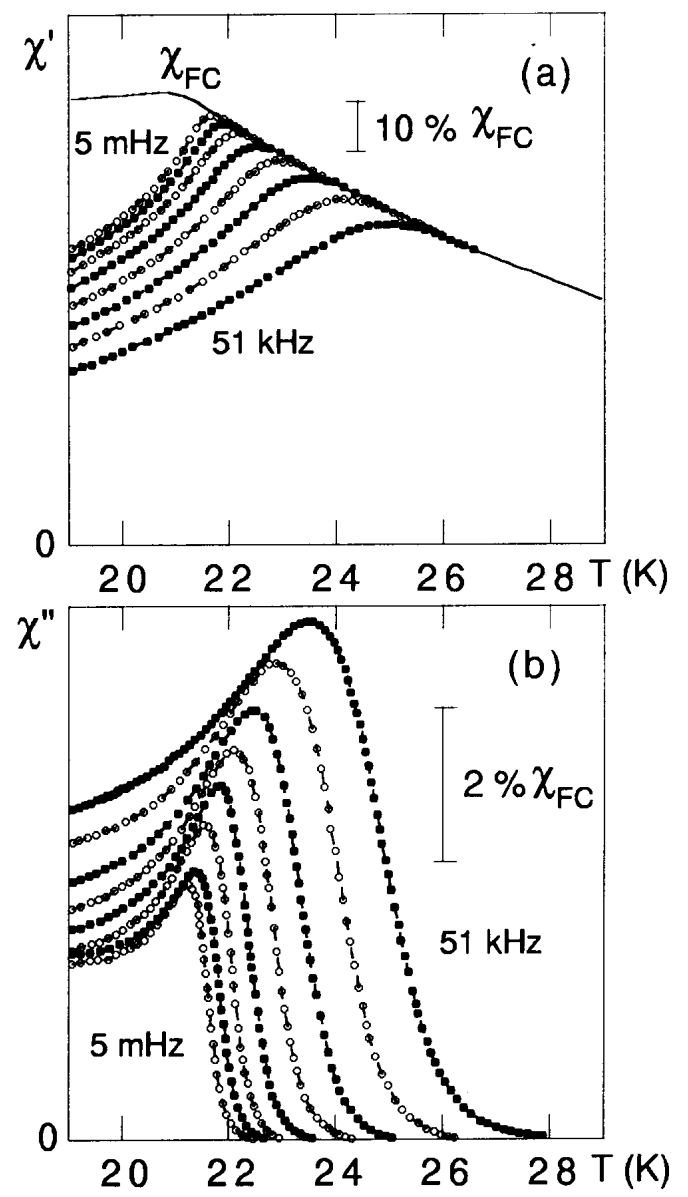

Figure 2. Ac susceptibility of $\mathrm{Fe}_{0.5} \mathrm{Mn}_{0.5} \mathrm{TiO}_{3}$ at $125 \mathrm{~Hz}$ in different superposed dc-fields varying from top to bottom as: $0,0.1,0.4,0.6,1,2$, and $3 \mathrm{~T}[6]$.
The conclusion from these dynamic and static scaling results is that the $3 \mathrm{~d}$ spin glass develops towards an equilibrium low temperature phase as the temperature is lowered towards $T_{g}$.

A more controversial problem is whether or not there is a spin glass phase in an applied magnetic field. Mean field solutions of the infinite range spin glass model say that there is a phase transition in a magnetic field, whereas the droplet model for 3d Ising spin glasses says no. Analytical work and some recent Monte Carlo (MC) simulations on short range 3d Ising spin glasses $[7,8]$ support the answer no, whereas other MC work is interpreted to favor an in-field phase transition in such systems [9].

What do experiments suggest? Fig. 3 shows constant relaxation time contours for our model system derived from ac susceptibility data in applied dc magnetic fields [6]. To extract information on whether a phase transition is anticipated or not on cooling in a field, we have analyzed the slowing down of the dynamics along the two lines drawn in Fig. 3. The result of various dynamic scaling analyses of the data is that the slowing down is best described by the following two scaling functions:

$$
\ln \left(\frac{\tau}{\tau_{0}}\right) \propto \Delta T_{f}^{-1}
$$

for $H=$ constant and

$$
\ln \left(\frac{\tau}{\tau_{0}}\right) \propto \Delta T_{f}^{-\left(1+\nu_{H} \Psi\right)}
$$

for $H / T=$ constant, where $T_{f}$ is the freezing temperature corresponding to each relaxation time and, in terms of the droplet model, $\Delta$ is an energy barrier, $\nu_{H}$ governs the field dependence of the correlation length and $\Psi$ is the barrier exponent. The result implies that there is no phase transition in a magnetic field for a realistic $3 \mathrm{~d}$ Ising spin glass. The exponent $\nu_{H} \Psi=0.65$, yielding $\Psi=0.8$ using a derived value of the exponent $\nu_{H}=0.8$. Experiments on a $3 \mathrm{~d}$ Ising model system thus support the scenario that there is a phase transition in zero field and no phase transition in a finite field, in accord with the droplet model prediction.

\section{Nonequilibrium dynamics}

In this section the nonequilibrium nature of the spin glass phase is discussed. Most experiments on this kind of dynamics have been performed on $3 \mathrm{~d}$ spin glass systems of less clear Ising nature than $\mathrm{Fe}_{0.5} \mathrm{Mn}_{0.5} \mathrm{TiO}_{3}$ and we have therefore chosen to exemplify nonequilibrium dynamics by measurements on other, but still characteristic, spin glass systems. The observed behavior is however also characteristic of $3 \mathrm{~d}$ Ising model systems as will be reported in future communications $[10]$. 


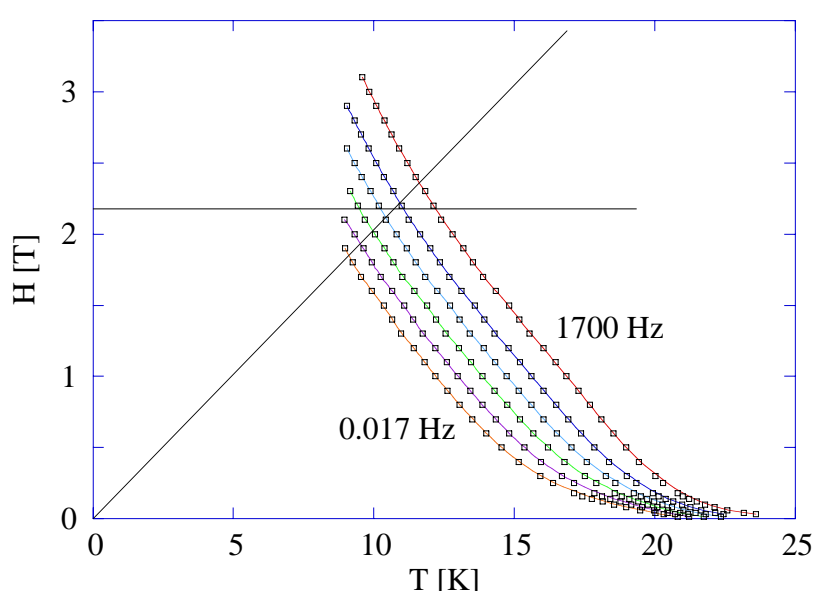

Figure 3. Constant relaxation time contours for $\mathrm{Fe}_{0.5} \mathrm{Mn}_{0.5} \mathrm{TiO}_{3}$ in an $\mathrm{H}-\mathrm{T}$ diagram. The frequences are $0.017,0.17,1.7,17,170$ and $1700 \mathrm{~Hz}$ and the corresponding relaxation times $t=1 / \omega[6]$.

An aging phenomenon in a magnetic system is revealed by a nonstationary response to the application of an external magnetic field, i.e., the response function depends on the waiting time $\left(t_{w}\right)$ spent at constant temperature before the magnetic field is applied. Fig. 4 shows results from zero field cooled (ZFC) magnetization measurements on a $3 \mathrm{~d}$ spin glass for different waiting times; (Fig. 4a) $m(t)$ vs. $\log (t)$ and (Fig. 4b) the corresponding relaxation rate $\left(S(t)=1 / h \frac{\partial m(t)}{\partial \ln (t)}\right)$. A striking change of the relaxation with $t_{w}$ is observed and the occurence of a maximum in $S(t)$ at an observation time of order the waiting time is characteristic for spin glasses. In an ac-susceptibility experiment, the observation time is constant $t=1 / \omega$ and at low frequencies aging can be observed as a spontaneous decay of both the in-phase and the out-of-phase component of the susceptibility with time at constant temperature. This behavior has been amply demonstrated in both $2 \mathrm{~d}$ and 3d spin glasses and also occurs in other frustrated magnetic systems [11].

The complexity of the nonequilibrium dynamics of spin glasses is further illustrated in Fig. 5, where the low frequency out-of-phase component of the acsusceptibility is plotted vs. temperature. The different curves have been recorded on continuous heating of the sample after different cooling procedures: continuous cooling (reference), cooling with one long stop at 40 $\mathrm{K}$ (single) and cooling with long stops at two different temperatures, 40 and $50 \mathrm{~K}$ (double) [12]. The dips in the curves occur at the temperatures where the sample has been kept at constant temperature during the otherwise continuous cooling process. Two crucial spin glass properties are illustrated by this figure: (i) chaos - equilibrating the sample at one specific temperature yields a spin configuration that appears random at a different temperature; the system is rejuvinated when the temperature is changed and (ii) memory - the equilibration that has been imposed on the system at a higher temperature is imprinted in the spin configura- tion and is retrieved when that temperature is recovered on heating. The combined memory and chaos behavior has been found to distinguish a spin glass phase from, e.g., a frustrated ferromagnetic phase [13] in which a rejuvination occurs both on heating and cooling, i.e., there is no memory effect.

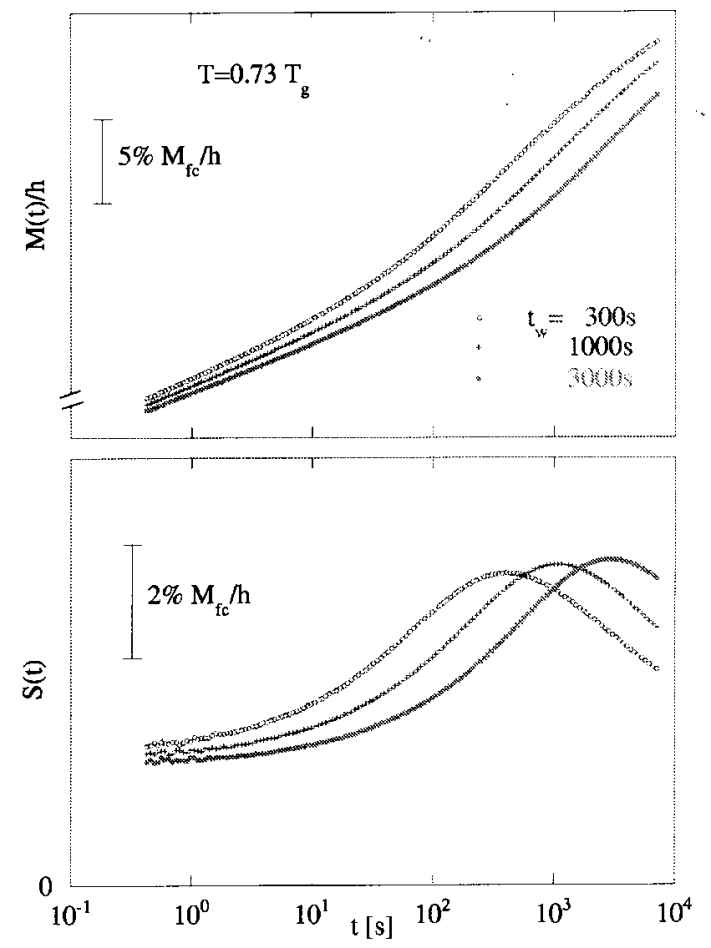

Figure 4. (a) Magnetisation vs. $\log (\mathrm{t})$ and (b) the corresponding relaxation rate (b) measured after different waiting times, $t_{w}$ for an amorphous metallic $3 \mathrm{~d}$ spin glass.

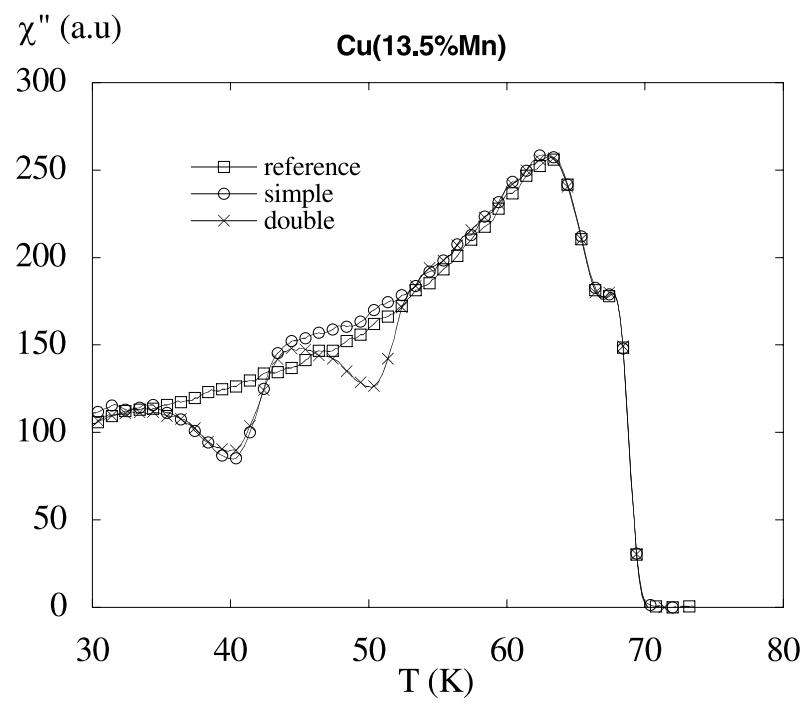

Figure 5. Low frequency $\chi^{\prime \prime}(T)$, measured on a $\mathrm{Cu}(\mathrm{Mn})$ spin glass on continuous heating after continuous cooling (ref), after one halt at $40 \mathrm{~K}$ (single) and after halting at two different temperatures, 40 and $50 \mathrm{~K}$, (double).

The chaos and memory behavior can be effectively demonstrated also in a simple dc magnetization vs. 
temperature experiment. Fig. 6 shows the ZFC, field cooled (FC) and thermoremanent (TRM) magnetization measured on a $\mathrm{Ag}(\mathrm{Mn})$ spin glass by cooling the sample to a low temperature in constant field and then applying/keeping/removing (ZFC/FC/TRM) a weak magnetic field and recording the magnetization on continuous heating, (open symbols) [14]. The curves marked by closed symbols have been recorded in the same way, the only difference being that the sample was kept at a constant temperature $T_{h}=27 \mathrm{~K}$ for some hours during cooling. There is a marked dip (bump) in the ZFC (TRM) curve in the region around $T_{h}$. This behavior again illustrates chaos and memory in the spin glass phase. (This simple experiment can be made on any high resolution magnetometer system to distinguish a spin glass phase from other disordered phases in less well understood disordered systems).

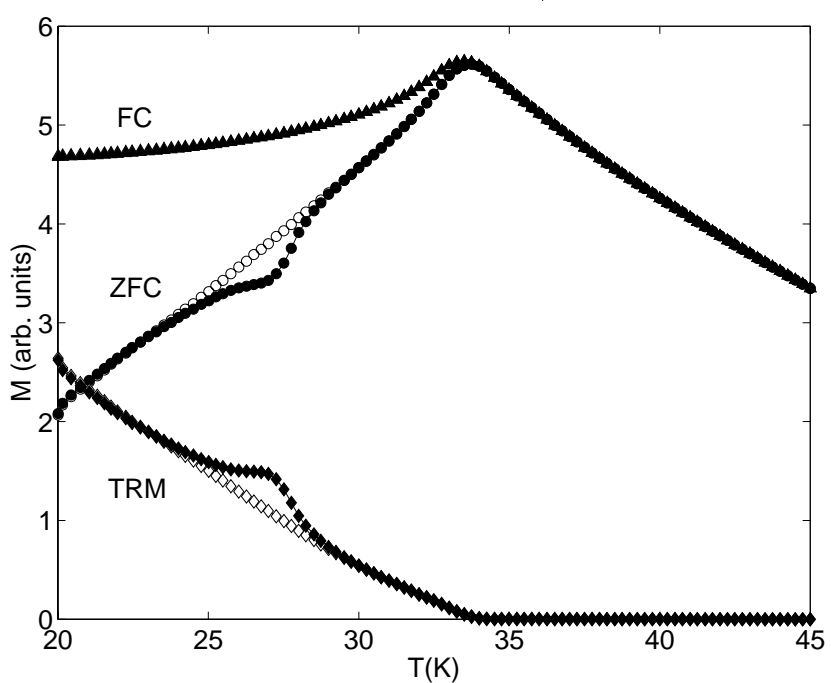

Figure 6. ZFC, FC and thermoremanent (TRM) measured on heating after cooling the $\mathrm{Ag}(\mathrm{Mn})$ spin glass to $20 \mathrm{~K}$ and then measuring on heating. The curves marked with full symbols represent a sample subjected to a long stop at constant temperature $(27 \mathrm{~K})$ and field during cooling [14].

\section{Discussion and conclusions}

In this paper we have recapitulated some key experimental properties of $3 \mathrm{~d}$ spin glasses. The observed behavior supports many features inherent to the droplet model of short-range $3 \mathrm{~d}$ spin glasses. The questions regarding the phase transition are answered in accord with this model: there exists a zero field phase transition, but there is no phase transition in a magnetic field.

Nonequilibrium dynamics, which in the droplet model is related to the growth of spin glass ordered regions, and the observed chaotic behavior also accord with the droplet picture. The memory phenomenon is a feature that perhaps can be incorporated in a droplet picture [15] or may require alternative approaches [12]. There are currently major efforts to understand the nonequilibrium and aging phenomena that disordered and frustrated physical systems possess [16].

\section{Acknowledgments}

Financial support from the Swedish Natural Science Research Council (NFR) is acknowledged.

\section{References}

[1] For recent reviews on spin glasses see Spin Glasses and Random Fields, A.P. Young, Ed., (World Scientific, Singapore, 1998).

[2] H. Aruga and A. Ito J. Phys. Soc. Jpn. 62, 4488 (1993).

[3] L. Lundgren, P. Svedlindh, P. Nordblad, and O. Beckman, Phys. Rev. Lett. 51, 911 (1983).

[4] K. Gunnarsson, P. Svedlindh, P. Nordblad, L. Lundgren, H. Aruga, and A. Ito, Phys. Rev. B 43, 8199 (1991).

[5] K. Gunnarsson, P. Svedlindh, P. Nordblad, L. Lundgren, H. Aruga, and A. Ito, Phys. Rev. Lett. 61, 754 (1988).

[6] J. Mattsson, T. Jonsson, P. Nordblad, H. Aruga Katori, and A.Ito, Phys. Rev. Lett. 74, 4305 (1995).

[7] See e.g., M. Palassini, and A. P. Young, Phys. Rev. Lett. 83, 5216 (1999); M. Palassini, and A. P. Young, condmat/0002134, and references therein.

[8] See e.g., M. A. Moore, H. Bokil, and B. Drossel, Phys. Rev. Lett. 81, 4252 (1998). B. Drossel, H. Bokil, and M.A. Moore, cond-mat/0007315, and references therein.

[9] See e.g., E. Marinari, G. Parisi, and J. J. Ruiz-Lorenzo, in Spin Glasses and Random Fields, A. P. Young, Ed., (World Scientific, Singapore, 1998), p. 59, and references therein.

[10] P. Jönsson et al., unpublished.

[11] P. Nordblad, in Dynamical Properties of Unconventional Magnetic Systems, A. T. Skjeltorp and D. Sherrington. Eds., (Kluwer, 1998), p. 343.

[12] K. Jonason, E. Vincent, J. Hammann, J. P. Bouchaud, and P. Nordblad, Phys. Rev. Lett. 81, 3243 (1998).

[13] E. Vincent, V. Dupuis, J. Hammann, M. Alba, and J. P. Bouchaud, Europhys. Lett. 50, 674 (2000).

[14] R. Mathieu, D. N. H. Nam, P. Jönsson, and P. Nordblad, cond-mat/0007070.

[15] T. Jonsson, K. Jonason, P. Jönsson, and P. Nordblad, Phys. Rev. B 59, 8770 (1999).

[16] J. P. Bouchaud, L. F. Cugliandolo, J. Kurchan, and M. Mézard M., in Spin Glasses and Random Fields, A. P. Young, Ed., (World Scientific, Singapore, 1998), p. 161. 